\title{
Etnoornitología guatusa
}

\section{Ginneth Pizarro Chacón}

Universidad Nacional, Costa Rica

La lengua guatusa ha sido objeto de estudios por parte de lingüistas que se han visto atraídos por ella. Las investigaciones realizadas se han dirigido a diversasáreas de la lingüística: los estudios sociolingüísticos y los estudios de lingüística descriptiva.

Por ejemplo, Betancourt y Constenla Umaña incursionan en la sociolingüística en "La expedición al territorio de los guatusos: una crónica colonial hispana y su contraparte en la tradición oral indíge-

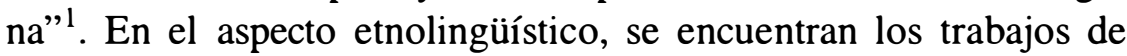
Constenla Umaña "Aspectos de la etnografía del habla de los guatusos", "El guatuso del palenque Margarita: su proceso de declinación" y "Hagiografía y antihagiografía en la tradición oral guatusa"2. Estas investigaciones contribuyen a identificarel guatuso comoparte integral de su comunidad, así como parte activa de la comunicación cotidiana.

Ligado a los estudios de lingüística descriptiva sobre la lengua, se encuentran, de Porras, El idioma guatuso: fonética y lexicografía y de Constenla Umaña, La lengua guatusa: fonología, gramática y léxico. Álvarez Navarro y otros (1979) aportan un Análisis fonológico y gramática generativo-transformacional del maleku (guatuso).

1. Helia Betancourt de Sánchez y Adolfo Constenla Umaña, "La expedición al territorio de los guatusos: una crónica colonial hispana y su contraparte en la tradición oral indígena", Revista de Filología y Lingüística de la Universidad de Costa Rica, VII, 1 y 2 (1981) 19-34.

2. Adolfo Constenla Umaña, "Aspectos de la etnografía del habla de los guatusos", Revista de Filología y Lingüística de la Universidad de Costa Rica, 1 (1982) 5-31; "El guatuso del palenque Margarita: su proceso de declinación”, Estudios de lingüística chibcha, VII (1988) 7-36; "Hagiografía y antihagiografía en la tradición oral guatusa", Revista de Filología y Lingüística de la Universidad de Costa Rica, XVIII, 1 (1992) 83-124. 
También podemos nombrar a Smith con "Un análisis fonológico del maleku", a Constenlacon "Descripción del sistema fonemático del guatuso" y, finalmente, a Sánchez con su artículo "Análisis fonológico

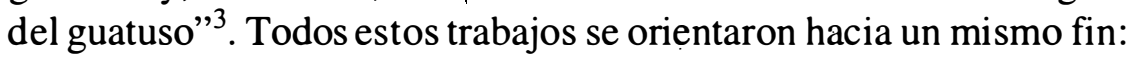
explorar y explicar de una manera clara y concisa lo relacionado con el sistema fonológico del guatuso.

Otro aspecto que atrajo la atención dentro de los estudios de linguística descriptiva es el área morfosintáctica. Entre las investigaciones realizadas podemos citar la de Sánchez, "El maleku: lengua ergativa" y las escritas por Constenla, "Sobre la construcción ergativa en la lengua guatusa" y "La voz antipasiva en guatuso"

Referente a estudios morfofonológicos, el único realizado hasta el momento es el de Constenla sobre "Morfofonología y morfología derivativa guatusas".5. Este mismo autor ha explorado el área de la lexicográfica en sus aportes acerca de la lexicosemántica del guatuso, "Construcción posesiva y economía morfoléxica en las lenguas del Área Colombiano-Centroamericana" y los nombres propios en esa lengua, que analiza en "Onomástica guatusa"

3. Álvaro Porras Ledezma, El idioma guatuso: fonética y lexicografía. Tesis de licenciatura (San José: Universidad de Costa Rica, 1959); Adolfo Constenla Umaña, La lengua guatusa: fonología, gramática y léxico. Tesis de licenciatura (San José: Universidad de Costa Rica, 1978); Emilia Álvarez Navarro y otros, Análisis fonológico y gramática generativo-transformacional del maleku (guatuso). Tesis de licenciatura (San José: Universidad de Costa Rica, 1979); Heidi Ṡmith, "Un análisis fonológico del maleku”, Revista de Filología y Lingüística de la Universidad de Costa Rica, V, 1 y 2 (1979) 31-54; Adolfo Constenla Umaña, "Descripción del sistema fonemático del guatuso", Revista de Filología y Lingüística de la Universidad de Costa Rica, IX, 1 (1983) 3-20; Víctor Sánchez, "Análisis fonológico del guatuso", Revista de Filología y Lingüística de la Universidad de Costa Rica, III (1984) 143-178.

4. Víctor Sánchez, "El maleku: lengua ergativa", Revista de Filología y Lingüística de la Universidad de Costa Rica, V, 1 y 2 (1979) 67-71. Los siguientes artículos de Adolfo Constenla Umaña: "Sobre la construcción ergativa en la lengua guatusa", Revista de Filología y Lingüística de la Universidad de Costa Rica, VIII, 1 y 2 (1982) 97-101 y "La voz antipasiva en guatuso", Revista de Filología y Lingüística de la Universidad de Costa Rica, V (1986) 85-96.

5. Adolfo Constenla Umaña, "Morfofonología y morfología derivativa guatusas", Revista de Filología y Lingüística de la Universidad de Costa Rica, IX (1990) 81-122.

6. Véanse de Constenla Umaña, "Construcción posesiva y economía morfoléxica en las lenguas del Área Colombiano-Centroamericana", Revista de Filología y Lingüística de la Universidad de Costa Rica, XI (1992) 101 -114 y "Onomástica guatusa", Revista de Filología y Lingüística de la Universidad de Costa Rica, XIV (1995) 41-88. 
Finalmente, en relación con el área etnopoética guatusa, debe citarse el estudio de Constenla, "Tres textos guatusos del ciclo narrativo de las uniones con los animales" y de Blanco, Castro y Constenla, La transformación de la tierra ${ }^{7}$.

En el plano de la descripción de biotaxonomías de lenguas indígenas de Costa Rica, mencionaremos el estudio de Edwards Mundall, "Etnoentomología cabécar" y otro realizado por Margery, "Etnoespantología cabécar"8.

No obstante los diversos estudios realizados sobre aspectos diferentes de esta lengua, no se encuentran trabajos en el plano de la etnosemántica que se refieran a biotaxonomías, ámbito que es propiamente el objeto del presente artículo, el cual expone en una forma precisa y detallada, la descripción taxonómica de las aves en guatuso ${ }^{9}$.

\section{Etnoornitología guatusa}

Tanto los nombres científicos como las respectivas denominaciones en español de todas las especies presentadas fueron tomadas de la taxonomía ornitológica expuesta por Stiles y Skutch ${ }^{10}$. En lo que a rasgos semánticos que forman el paradigma se refiere, su determinación es producto de la cultura guatusa y de la observación y conocimiento general del informante.

\subsection{El principio único}

La etnoornitología guatusa muestra un ordenamiento piramidal en el que se da un orden de mayor a menor inclusividad. Desde este

7. Adolfo Constenla Umaña, "Tres textos guatusos del ciclo narrativo de las uniones con los animales", Revista de Filología y Lingüística de la Universidad de Costa Rica, X (1991) 101 120; Antonio Blanco Rodríguez, Eustaquio Castro Calvo y Adolfo Constenla Umaña, La Klá majifijicá (La transformación de la tierra) (San José: Editorial de la Universidad de Costa Rica, 1993) 62-64.

8. Enrique Margery Peña, "Etnoespantología cabécar", Revista de Filología y Lingüistica de lı Universidad de Costa Rica, XII, 1 (1986) 153-188.

9. Se trata de las conclusiones de un estudio más amplio, Ginneth Pizarro, Etnoornitología guatusa. Tesis de Maestría en Lingüística (San José: Universidad de Costa Rica, 1998).

10. Gary Stiles y Alexander F. Skutch, Guía de aves de Costa Rica (Heredia: InBio, 1995). 
punto de vista, el taxón de mayor inclusividad es el principio único, representado por la expresión orróqui maráma equivalente al término ('cosas que tienen vida'). Este principio único comprende, a su vez, el taxón irifáque maráma ('cosas que tienen vida no humana-aves') y el taxón acanhe maráma ('cosas que tienen vida pero son manejadas por el hombre-carro, avión'). Un rasgo común a los irifáque maráma y que los hace orróqui maráma es el hecho de ser túru cónhe ('cosa del monte').

\section{2. La forma de vida mayor}

$\mathrm{Al}$ referimos a la forma de vida mayor, denotamos un taxón de mayor inclusividad en el estudio taxonómico de una lengua dada. El taxón de mayor inclusividad en el universo ornitológico del guatuso es representado porel lexema maráfáque sufá maráma, que corresponde a 'voladores'.

Al analizar el plano paradigmático, es de importancia recalcar que la forma de vida mayor implica la inclusión de uno o más rasgos comunes que identifican el dominio semántico, lo cual es base fundamental del paradigma.

En este caso, la forma de vida mayor abarca tres rasgos comunes a todas las aves en guatuso: curúcurú tenénhe ifilhí ('el ojo es redondo'); epéme icóri líca anh ('no tiene leche') y maráfáque sufá (maráma) ('voladores').

La aparición de estos tres rasgos comunes a todas las aves se da en un ámbito general y excluyente. En el ámbito general, estos son rasgos primarios obligatorios a todos los genéricos, desde taxas polisémicas con una o más especies, hasta aquellas taxas particulares al resto. La presencia de estas, aunada a los rasgos particulares de cada genérico, lo convierte en parte de una forma de vida ornitológica.

En cuanto al valor excluyente, estos tres rasgos discriminan a este grupo de cualquier otra forma de vida mayor existente, dando como resultado un denominadornegativo al resto de las especies. Además, estos rasgos crean particularidad en el análisis taxonómico realizado, al atribuir características propias a un grupo determinado de especies. 
1.3. Determinación y caracterización de los nombres genéricos

$\mathrm{Al}$ referimos a los nombres genéricos, se hace hincapié en las taxas que designan los géneros, es decir, las taxas que tienen una menor inclusividad que la designada por la forma de vida mayor. Estos genéricos designan la posesión de los tres rasgos de vida mayor y uno o más rasgos adicionales. Cada uno de estos genéricos reúne las características requeridas para constituir la definición componencial de un término, siendo esto lo equivalente a su significatum.

Partiendo de lo anterior, el mundo omitológico del guatuso está compuesto por cincuenta y cinco taxas genéricas. A continuación se detallan los nombres correspondientes a cada genérico en el idioma guatuso, especificándose la familia de la taxonomía científica a la cual pertenecen y su correspondiente en español:

i. táro

ii. macmac

iii. chólechóle

iv. pílil

v. túli

vi. túfi

vii. pocóronh

viii. úrro especies de anatidae, podicipedidae, heliomithidae (patos);

especies de ardeidae (cuaca);

especies de anhingidae, phalacrocoracidae (patos);

especies de anatidae, jacanidae, scolopacidae (gallito de agua);

especies de columbidae (palomas);

especies de cracidae (pavón);

especies de phasianidae (chirrascuá, codorniz);

especies de cathartidae (buitre, zopilote); 
ix. nhualhquic

x. móra

xi. cosóqui

xii. arárafá

xiii. mucmuc

xiv. téco

xv. culhímoquí

xvi. corrémecmec

xvii. nhuáco

xviii. panhá ó

xix. lhijílhaífa

xx. concuilhcuilh

xxi. píli especies de accipitridae (gavilán sabanero);

especies de accipitridae (águila arpía); especies de psittacidae (lora, zapoyol); especies de strigidae (búho); especies de strigidae, tytonidae (lechuza);

especies de cuculidae (cuclillos);

especies de caprimulgidae (cuyeo, pucuyo);

especies de galbulidae, momotidae (jacamares y momotos);

especies de falconidae (guaco);

especies de accipitridae, falconidae (gavilán pequeño);

especies de accipitridae, falconidae (gavilán, aguilucho);

especies de accipitridae (tipo de gavilán);

especies de ramphastidae (tucanes); 
xxii. pilucpilunh

xxiii. carécar

xxiv. chóchófa

xxv. lhorrórro

xxvi. óla

xxvii. chacárra

xxviii. cútu

xxix. quilhanhquilh

xxx. nhoronh

xxxi. ujúcotenhcot

xxxii. charáraf

xxxiii. jamnaráre

xxxiv. caélocaélo especies de tyranidae (tijerilla, mosquero);

especies de alcedinidae (martines pescadores);

'especies de picidae, nyctibiidae, furnariida (carpinteros);

especies de picidae (carpinteros);

especies de psittacidae (lapa roja);

especies de gallinae (gallinas);

especies de chiropterae (murciélagos);

especies de ciconiidae, ardeidae (galán sin ventura);

especies de threskiornithidae, ciconiidae (coquito, guairón);

especies de rallidae (poponé, ponepone);

especies de rallidae, formicariidae (charrarra);

especies de burhinidae (alcaraván); especies de cracidae (chachalaca); 
XXXv. úru

xxxvi. Ihúlu

xxxvii. tónhor

xxxviii. lhúrirren

xxxix. chólhi

xl. canhánhar

xli. tóji cuacsuf

xlii. cácáfa

xliii. tójisuífa ó

xliv. tóji lhifúru

xlv. tóji arára

xlvi. lhinhcu

xlvii. tí cuacsuf

xlviii. lhuélhuéfa especies de cracidae (pava);

especies de tinamidae (gongolona);

especies de tinamidae (perdiz)

especies de tinamidae (yerre);

especies de phasianidae (codorniz);

especies de accipitridae (aguilucho);

especies de accipitridae (gavilán);

especies de accipitridae (gavilán alicastaño);

especies de accipitridae (gavilán);

especies de falconidae (gavilán cacao);

especies de falconidae, accipitridae (gavilán);

especies de accipitridae (gavilán tijera);

especies de accipitridae (gavilán sabanero);

especies de pandionidae (gavilán pescador); 
xlix. mulhtutúcu

especies de columbidae (tórtolas o espíritu santo);

l. querrácaquerráca especies de psittacidae (chucuyo);

li. cumáso

especies de caprimulgidae (cuyeos);

lii. Ihaláqui cosonh

especies de trogonidae (quetzal);

liii. cajanh

especies de trogonidae (trogones);

liv. lhújo

especies de icteridae (oropéndola);

lv. nhiscacá

Este último grupo está formado por varias especies, tales como ticarápanh / especies de capitonidae, furnariidae, cotingidae (cocora, piricuaco); chásirrúja / especies de tyrannidae (mosqueros); toréretorére / especies de fumariidae (tirahojas barbiescamado); chicháúnha / especies de thraupidae, formicariidae, icteridae (sargentos); tuílhirrírri / especies de furnariidae, tyrannidae (trepamusgos); lhurílhurí / especies de apodidae (vencejos y golondrinas); pilhí / especies de apodidae, trochilidae (macúa, colibríes); sarrásarr / especies de formicariidae, cotingidae, parulidae (hormigueros); péti / especies de formicariidae, fumariidae, vireonidae, parulidae, sylviidae, tyrannidae, bucconidae (hormigueros); pintot / especies de icteridae (chorcha); curíyocuríyo / especies de formicariidae, parulidae (batará grande, candelita norteña); tecteccarája / especies de bucconidae, tyrannidae, thraupidae (mosqueros); purúnhuénhué / especies de momotidae (viuditos); úrifirrunh / especies de formicariidae (hormiguero ocelado); querérequerére / especies de capitonidae (viudito); pocpoquíífa / especies de scolopacidae, corvidae, icteridae (piapias); quirríchicchic / especies de bucconidae, furnariidae, thraupidae 
(soterrecillo, tángara); pirrunh / especies de furnariidae, formicariidae (hormigueros); chulhpa ó / especies de formicariidae, cotingidae, tyrannidae (reinitas); tí sulí / especies de charadriidae (chorlito); tarrchíyonh / especies de pipridae (toledo); tocórro (óra) / especies de troglodytidae, turdidae (soterrés y zorzales); quirrictayonh / especies de turdidae (yigüirro); Ihuí (óra) / especies de turdidae, bombycillidae (jilguero); chióqui (óra) / especies de emberizidae (arroceros).

\subsection{Los rasgos adicionales}

Dentro del paradigma se presenta una dimensión semántica comprendida por veintidós rasgos, los cuales fundamentan el análisis componencial de las taxas de los genéricos. Doce de estos corresponden a rasgos morfológicos y el resto a no morfológicos de naturaleza perceptual y cultural por parte del informante.

De estas variables, algunas son de expresión binaria, en tanto que otras corresponden a taxonomías múltiples. Entre estas taxonomías múltiples se han incluido los rasgos ala, pico, pata, colores, hábitat, morfogénesis, vuelo, canto, hora de aparición y alimentación, por tener características dentro de su propio microparadigma.

En estos veintidós rasgos configurados en el paradigma, cada variable está dividida en subvariables relativas a su respectivo rasgo. Estas subvariables clarifican la disposición de los rasgos necesarios y suficientes para la definición componencial de cada taxa.

A continuación se enumeran estos rasgos adicionales, detallando los morfológicos y sus respectivas subvariables, en su relación con las taxas analizadas y rasgos de expresión binaria, en algunos de los casos.

\subsubsection{La variable longitud del ala}

La primera variable de la dimensión semántica del paradigma denota el rasgo morfológico ala, al cual corresponden dos rasgos morfológicos que se expresan por la oposición +/-, donde lharátenh ('larga') denota el cualificador positivo oponiéndose al operador negativo que indica lhacá tené ('corta'). Este rasgo, a su vez, está 
formado por tres rasgos no morfológicos, tales como, síjafa icuinhca rriuránhe ('mueve el ala despacio'), llevando este la connotación positiva, opuesto a ojá tené icuinhca rriuránhe ('mueve el ala rápido'), el cual funciona como el operador negativo. El tercer rasgo no morfológico es la subvariable ácafá icuinhca ripáye ('el ala le suena raro'), el cual es denotado con un marcador positivo. Estas últimas subvariables son atribuidas a cualidades perceptivas del informante y no a algo tangible o visible para el investigador.

\subsubsection{La variable forma del pico}

La segunda variable, también de tipo morfológico, se refiere a la forma del pico. Esta variable se manifiesta en una taxonomía múltiple, integrada por cinco rasgos: iocá lharátenh ('pico largo'), donde un operador negativo muestra la característica iocá nocóchánhe ('pico corto'). Las tres últimas subvariables hacen referencia a iocá pilíchu inhánhe ('pico puntiagudo'), iocá suíyanh ('pico curvo') y iocá talátenh ('pico ancho').

\subsubsection{La variable longitud de las patas}

Esta tercera variable, también morfológica, está formada por siete subvariables, en donde las dos primeras se denotan por la oposición+/-ilhóqui chá ámpenh ('pata grande'), siendo el operador negativo representado por ilhóqui chá nocóchánhe ('pata pequeña'). La tercera subvariable hace referencia a una comparación con otro animal, ilhóqui chá chacárra inhánhe ('pata como de gallina'). Una cuarta subvariable es ilhóqui chá talátenh ('pata plana'), que es denotada con el operador positivo. La quinta y sexta subvariables se expresan por la oposición +/- ilhóqui chá lharátenh ('pata larga'), en la que el término negativo implica el atributo ilhóqui chá lhacá tenénhe ('pata corta'). Finalmente, la sétima subvariable, ampenhé ilhóqui lenh ('la uña es grande / garra') muestra el atributo positivo dentro del paradigma. 
1.4.4. La variable forma y longitud de las orejas

La cuarta variable, de tipo morfológico. está compuesta por tres rasgos: los dos primeros se expresan en una oposición $+/-$, donde nicaní itocótocó lharátenh ('oreja larga') lleva la connotación positiva, mientras que su operador negativo es denotado por el atributo itocótocó lhacá tenénhe ('oreja corta'). El tercer rasgo, itocótocó pilíchu inhánhe ('oreja puntiaguda'). se muestra bajo el denominador positivo.

\subsubsection{La variable posesión de barba}

La quinta variable, correspondiente a un atributo morfológico. presenta rasgos de taxonomía binaria. Estos son manifestados en una oposición +/- iconhílha anh ('tiene barba'). en la que el operador negativo implica el rasgo epéme iconhílha anh ('no tiene barba').

\subsubsection{La variable longitud de la cola}

Esta sexta variable. también de tipo morfológico. denota una taxonomía binaria por medio de la oposición +/-. en la cual el atributo positivo es manifestado por el rasgo ituquí ampenh ('cola larga / grande') y su correspondiente negativo lo denota el atributo ituquí lhacá tenénhe ('cola corta').

\subsubsection{La variable tamaño y forma de los ojos}

La sétima variable. de origen morfológico, presenta una taxonomía múltiple representada por tres rasgos: los dos primeros muestran una oposición +/- ampenhé ifilhi ('ojos grandes'). en la que el operador negativo denota el rasgo atributivo nocóchánhe óra ifilhi ('ojos pequeños'). El tercer rasgo curúcurú tenénhe ifilhí ('ojos redondos') representa el atributo positivo.

\subsubsection{La variable posesión de cresta}

Esta octava variable, correspondiente a un atributo morfológico. muestra una taxonomía binaria expresada por la oposición +/- ilhorrórro anh ('tiene cresta'), en la que el término negativo conlleva la característica epéme ilhorrórro ('no tiene cresta'). 


\subsubsection{La variable longitud del pescuezo}

La novena variable, de tipo morfológico, también se expresa binariamente con la oposición + /- ichá quírra lharátenh ('pescuezo largo'), cuyo operador negativo corresponde al atributo ichá quírra lhacátenénhe ('pescuezo corto').

\subsubsection{La variable tipo de color y posesión de manchas}

Esta décima variable, correspondiente a un atributo morfológico, se manifiesta en una taxonomía múltiple, formada por once rasgos. Los siete primeros hacen referencia al color que poseen los diferentes tipos de especies analizadas. Dentro de este grupo se muestran uchúli inhánhe ('negro'), lí inhánhe ('rojo'), lhanhánhe ('verde'), taclhará inhánhe ('café'), pórilhá lhútu inhánhe ('amarillo'), jaríjaríyanh ('veteado'), imlequícanh ('blanco'). Los siguientes tres rasgos denotan la aparición o no de manchas. Así, bajo el rasgo positivo se presentan los rasgos ipurúco riat ('manchas en el cuerpo'), ilhoctocóra riat ('manchas en el pecho'), itunhco riat ('manchas en la cola'). Finalmente, la posesión de un color en una parte específica del cuerpo es mostrada por el atributo positivo ilhoctocóra porílha lhútu inhánhe ('pecho amarillo').

\subsubsection{La variable tamaño}

La decimoprimera variable, la última correspondiente a un atributo morfológico, se manifiesta en una taxonomía binaria expresada por la oposición +/- tamaño ampenh ('tamaño grande'), cuyo operador negativo muestra el atributo tamaño nocóchánhe ('tamaño pequeño').

\subsubsection{La variable morfogénesis}

La decimosegunda variable, que se asocia a la forma del nacimiento, se representa con la oposición +/- ipúco ifáparr ('nace por huevo'), y su operador negativo hace referencia al atributo epéme ipúco ifáparr ( 'no nace por huevo'). El único genérico que es cubierto por el término marcado de la oposición es cútu ('murciélago'). Es de 
interés señalar un rasgo atributivo positivo que hace referencia al lugar donde se deposita el huevo, córa nocóchánhe óra ipú rriarr ( 'pone el huevo en árbol pequeño').

\subsubsection{La variable hábitat}

La decimotercera variable se asocia con el lugar de morada o reposo y está representada por una taxonomía múltiple, formada por los siguientes rasgos: córalhá ipunh ('vive en árbol'), córa nocóchánhe óra cuiclha ipunh ('vive en ramas de árbol pequeño'), córa cuiclha ichújiy ('duerme en las ramas del árbol'), córa nocóchánhe óralhá ipunh ('vive en árbol pequeño'), turúco ipunh ('vive en la montaña'), córa cháco ipunh ('vive en la copa del árbol'), córa cuiclha ipunh ('vive en la rama del árbol'), córa cuiclha iaréje rriquerr ('anida en rama del árbol'), córa nocóchanhe óralhá iaréje rriquerr ('anida en árbol pequeño'), córa calíco ipunh ('vive en hueco del árbol'), tí jólalhá ipunh ('vive en la orilla del río'), lacá calíco ipunh ('vive en hueco en la tierra'), córa nocóchanhe óra cuiclha iaréje rriquerr ('anida en rama de árbol pequeño'), lacá calíco iúpal ('duerme en cueva'), típala ipunh ('vive en el estero'), lácalha ipunh ('vivee nel suelo') y fuéja ipunh ('vive en la maleza').

Dos rasgos importantes y parte de esta variable son los atributos positivos córa purúco iú ('parado sobre la corteza del árbol'), así comoel rasgo córa rrifolóje ('pica el árbol'). Porúltimo, se considera un rasgo que tiene que ver con el no contacto con el suelo; este es denotado por el rasgo positivo epéme lacálha iafáre ('no toca el suelo').

\subsubsection{La variable tipo de vuelo}

Esta decimocuarta variable, relacionada con la altura del vuelo o a la no existencia de este, está constituida por cuatro rasgos: lacánifá rifáque ('vuela bajo'), ujúruní rifáque ('vuela alto'), tójicuinhni rifáque ('vuela altísimo') y epéme rifáque ('no vuela'). 
1.4.15. La variable capacidad de cantar

La decimoquinta variable se manifiesta por la oposición +/iporétenh ('canta'), y su correspondiente operador negativo lo constituye la característica epéme iporétenh ('no canta'). Dentro de esta variable se incluye el atributo positivo maráláfarinháfa iporétenh ('canta bonito').

\subsubsection{La variable altura del canto}

La decimosexta variable y segunda correspondiente a la variable canto se expresa en la oposición +/- capífa iporétenh ('canta duro'), y su término negativo representado por el atributo epéme capífa iporétenh ('no canta duro'). Dentro de esta subvariable se denota un rasgo positivo atribuible al alcance de dicho canto iporétecá carrtaúni ripáye ('cantan y se escucha a lo lejos'). Además, algunos se diferencian iporétenh qúa ('por su canto').

\subsubsection{La variable hora del canto}

Esta decimosétima variable y tercera de la variable canto está subdividida en tres rasgos: cumáco iporétenh ('canta temprano'), tóji tuéta iporétenh ('canta al atardecer') y tiúricá iporétenh ('canta por la noche').

\subsubsection{La variable hora de aparición}

La decimoctava variable, asociada con la hora de salida o aparición de dichas especies, es de un tipo de taxonomía múltiple. Esta se divide en tres rasgos: ipítenhé tójic ('sale de día'), ipítenhé tóji tuéta ('sale al atardecer') y ifánhe tiúric ('anda de noche').

\subsubsection{La variable fagoica}

Esta decimonovena variable se relaciona con el tipo de alimento consumido por las diferentes especies analizadas y que el informantc y su cultura vernacular consideran como característicos de dichos genéricos. Esta variable está constituida por una taxonomía muilliplc 
formada por los siguientes rasgos: carráqui rricanh ('come gusanos'), quilhúqui ('saltamontes'), calhíjaqui ('garrapatas'), lhúma ('pejibaye'), culhá ('ardilla'), tiú ('mono'), lherrélherréjanhé rrilanh ('come carroña'), mulhú óra ('pescaditos'), córa cúru ('fruta'), nhiscacá óra ('pajaritos'), lhaláqui ('culebra'), cajú ('caco'), aíqui ('maíz'), orróqui lhútu cólalí rrichie ('néctar de la flor'), lucúlucú óra ('sapitos'), orróqui ampenh ('animales grandes'), chiquitarequi cúru áunh ('hoja de frijol'), úlenh ('lagartija'), lhunhóqui cúru ('arroz'), lhánhá ('banano'), lhúla únha ('banano maduro'), líchuchúsu ('chupa / toma sangre'). Se adjuntan dos rasgos atributivos positivos que hacen referencia al hecho de aquel genérico que se ichá rriulúlunh ('se zambulle en el río') y aquel que tíco ipsirínhe ('pesca en el río').

\subsubsection{La variable dar vuelta a la cabeza}

La vigésima variable se relaciona con la propiedad de poder o no mover su cabeza en una forma circular. Este rasgo es de un tipo de taxonomía binaria y está constituido por una oposición +/- ichá rricoróronh ('dar vuelta a la cabeza'), cuyo operador negativo es representado por el atributo epéme ichá rricoróronh ('no da vuelta a la cabeza').

\subsubsection{La variable comestible}

Esta variable se asocia con el hecho de consumo de estos genéricos por parte de dicha cultura. También se manifiesta como una taxonomía binaria representada por una oposición +/- malac ('es comida'), y su término negativo denotado por la característica epéme malac ('no es comida').

\subsubsection{La variable comportamiento}

Hace referencia a las especies comprendidas por cada genérico que muestran un comportamiento peculiar hacia los seres humanos. Se manifiesta una taxonomía múltiple constituida por cuatro rasgos: 
iunhé foctenhé ótaca marrícuánhe ('se ponen inquietos cuando nos ven'), epéme iunhé foctenhé ótaca marrícuánhe ('no se ponen inquietos cuando ven gente'), matuclha mayú óje ('nos persiguen') y epéme matuclha óje ('no nos persiguen').

\section{Definiciones componenciales de los nombres genéricos}

El siguiente análisis muestra una definición componencial de algunos de los nombres genéricos mencionados en 1.3. En esta definición componencial, se suscriben los tres primeros rasgos comunes a todas las especies de aves en la forma de vida mayor, denotados con las abreviaturas (R.C.), expuestos en 1.2. Estos abarcan: curú curú tenénhe ifilhi ('ojos redondos'), épeme i córi líca anh ( 'no tiene leche') y maráfáque sufá (maráma) ('voladores'), además de los rasgos adicionales a cada genérico. Se adjunta, a cada rasgo del análisis componencial en guatuso, su correspondiente en español para un mejor entendimiento de cada uno de los genéricos y sus específicos típicos y no típicos. en caso de que los tengan.

(i) táro (anatidae, podicipedidae, heliornithidae) ('patos')

Este genérico es polisémico con un específico típico y un específico no típico. Este genérico presenta los siguientes rasgos: R.C. + lhacá tené icuinhca ('alas cortas'), síjafá icuinhca rriuránhe ('mueve el ala despacio'), ácafá icuinhca ripáye ('hace ruido al volar'), Iharátenh iocá ('pico largo'), iocá suíyanh ('pico curvo'), iocá talátenh ('pico ancho'), ilhóqui chá ámpenh ('pata grande'), lhacá tenénhe ilhóqui chá ('pata corta'), ilhóqui chá talátenh ('pata plana'), ituquí lhacá tenénhe ('cola corta'), nocóchánhe óra ifilhi ('ojos pequeños'), ichá quírra lharátenh ('pescuezo largo'), uchúli inhánhe ('negro'), córa cuiclha ipunh ('vive en la rama del árbol'), córa nocóchánhe óra ipú rriarr ('pone el huevo en árbol pequeño'), lacálha iafáre ('toca suelo'), ipúco ifáparr ('nace por huevo'), tójicuinhni rifáque ('vuela altísimo'), ipítenhé tójic ('sale de día'), 
ipítenhé tóji tuéta ('sale al atardecer'), mulhú óra rricanh ('come pescaditos'), ichá rriulúlunh ('se zambulle'), tíco ipsirínhe ('pesca en el río'), malac ('comestible').

Las especies dentro de este genérico comparten los mismos rasgos de este, más otros que los distinguen de los de su mismo grupo. Se detallarán el o los rasgos diferenciadores que hacen que el específico no típico se diferencie del genérico. Este específico no típico es:

Táro (suírra) ('pato real'): turúco ipunh ('vive en la montaña').

(ii) macmac (ardeidae) ('cuaca')

Este genérico presenta un específico típico cuyo denominador es mac mac, polisémico en relación con el genérico y posee los siguientes rasgos: R.C. + lhacá tené icuinhca ('alas cortas'), síjafá icuinhca rriuránhe ('mueve el ala despacio'), lharátenh iocá ('pico largo'), iocá suíyanh ('pico curvo'), iocá talátenh ('pico ancho'), ilhóqui chá ámpenh ('pata grande'), lhacá tenénhe ilhóqui chá ('pata corta'), ilhóqui chá talátenh ('pata plana'), ituquí lhacá tenénhe ('cola corta'), nocóchánhe óra ifilhi ('ojos pequeños'), ichá quírra lharátenh ('pescuezo largo'), uchúli inhánhe ('negro'), córa cuiclha ipunh ('vive en la rama del árbol'), córa nocóchánhe óra ipú rriarr ('pone el huevo en árbol pequeño'), lacálha iafáre ('toca suelo'), ipúco ifáparr ('nace por huevo'), lacánifá rifáque ('vuela bajo'), ipítenhé tójic ('sale de día'), ipítenhé tóji tuéta ('sale al atardecer'), tiúric ifánhe ('anda de noche'), mulhú óra rricanh ('come pescaditos'), ichá rriulúlunh ('se zambulle'), tíco ipsirínhe ('pesca en el río').

(iii) chóle-chóle (anhingidae, phalacrocoracidae) ('patos')

Este genérico presenta un específico típico, cuyo denominador es chóle chóle, polisémico en relación con el genérico y posee los siguientes rasgos: R.C. + lharátenh icuinhca ('alas largas'), síjafá icuinhca rriuránhe ('mueve el ala despacio'), iocá nocóchánhe ('pico corto'), iocá suíyanh ('pico curvo'), iocá talátenh ('pico 
ancho'), ilhóqui chá ámpenh ('pata grande'), lhacá tenénhe ilhóqui chá ('pata corta'), ilhóqui chá talátenh ('pata plana'), ituquí lhacá tenénhe ('cola corta'), nocóchánhe óra ifilhi ('ojos pequeños'), ichá quírra lharátenh ('pescuezo largo'), uchúli inhánhe ('negro'), córa cuiclha ipunh ('vive en la rama del árbol'), córa nocóchánhe óra ipú rriarr ('pone el huevo en árbol pequeño'), lacálha iafáre ('toca suelo'), ipúco ifáparr ('nace por huevo'), lacánifá rifáque ('vuela bajo'), ipítenhé tójic ('sale de día'), mulhú óra rricanh ('come pescaditos'), ichá rriulúlunh ('se zambulle'), tíco ipsirínhe ('pesca en el río').

(iv) pílil(anatidae, jacanidae, scolopacidae) ('gallito de agua')

Este genérico presenta un específico típico, cuyo denominador es pilil, polisémico en relación con el genérico y posee los siguientes rasgos: R.C. + lhacá tené icuinhca ('alas cortas'), síjafá icuinhca rriuránhe ('mueve el ala despacio'), iocá lharátenh ('pico largo'), iocá suíyanh ('pico curvo'), iocá talátenh ('pico ancho'), ilhóqui chá ámpenh ('pata grande'), lhacá tenénhe ilhóqui chá ('pata corta'), ituquí lhacá tenénhe ('cola corta'), nocóchánhe óra ifilhi ('ojos pequeños'), ichá quírra lhacátenénhe ('pescuezo corto'), lhanhánhe ('verde'), córa cuiclha ipunh ('vive en la rama del árbol'), córa nocóchánhe óra ipú rriarr ( 'pone huevo en árbol pequeño'), lacálha iafáre ('toca suelo'), ipúco ifáparr ('nace por huevo'), lacánifá rifáque ('vuela bajo'), ipítenhé tójic ('sale de día'), mulhú óra rricanh ('come pescaditos'), ichá rriulúlunh ('se zambulle'), tíco ipsirínhe ('pesca en el río').

(v) túli (columbidae) ('palomas')

Este genérico es polisémico con un específico típico y tres específicos no típicos. Presenta los siguientes rasgos: R.C. + lhacá tené icuinhca ('alas cortas'), síjafá icuinhca rriuránhe ('mueve el ala despacio'), iocá nocóchánhe ('pico corto'), ilhóqui chá nocóchánhe ('pata pequeña'), lhacá tenénhe ilhóqui chá ('pata corta'), 
ilhóqui chá chacárra inhánhe ('pata como de gallina'), ituquí lhacá tenénhe ('cola corta'), nocóchánhe óra ifilhi ('ojos pequeños'), ichá quírra lhacátenénhe ('pescuezo corto'), uchúli inhánhe ('negro'), córa nocóchánhe óralhá ipunh ('vive en árbol pequeño'), córa nocóchánhe óra ipú rriarr ('pone huevo en árbol pequeño'), lacálha iafáre ('toca suelo'), ipúco ifáparr ('nace por huevo'), lacánifá rifáque ('vuela bajo'), iporétenh ('canta'), maráláfarinháfa iporétenh ('canta bonito'), cumáco iporétenh ('canta temprano'), tóji tuéta iporétenh ('canta al atardecer'), ipítenhé tójic ('sale de día'), ipítenhé tóji tuéta ('sale al atardecer'), lhúma/córa cúru rricanh ('come pejibayes / frutas'), malac ('comestible').

Las especies dentro de este genérico comparten los mismos rasgos de este, más otros que los diferencian de los de su mismo grupo. Se detallarán el o los rasgos diferenciadores que hacen que los específicos no típicos se distingan entre sí.

Un primer específico no típico es:

Caracche túli ('tortolita, paloma'): pórilhá lhútu inhánhe ('amarillo').

Un segundo específico no típico es:

Tafá túli ('paloma'): lí inhánhe ifilhí ('ojos rojos').

Un tercer específico no típico es:

Cunh-óra/cunh ('paloma'): nocóchánhe ('pequeño'), iporétenh qúa ('por su canto').

(vi) túfi (cracidae) ('pavón')

Este genérico presenta un específico típico, cuyo denominador es túfi, polisémico en relación con el genérico y posee los siguientes rasgos: R.C. + lharátenh icuinhca ('alas largas'), síjafá icuinhca rriuránhe ('mueve el ala despacio'), ácafá icuinhca ripáye ('hace ruido al volar'), iocá nocóchánhe ('pico corto'), iocá suíyanh ('pico curvo'), ilhóqui chá ámpenh ('pata grande'), ilhóqui chá lharátenh ('pata larga'), ilhóqui chá chacárra inhánhe ('pata como de gallina'), ituquí ampenh ('cola larga'), nocóchánhe óra ifilhi ('ojos 
pequeños'), ilhorrórro anh ('tiene cresta'), ichá quírra lhacátenénhe ('pescuezo corto'), uchúli inhánhe ('negro'). ilhoctocóra riat ('manchas en el pecho'), córa cuiclha ipunh ('vive en la rama del árbol'), córa nocóchánhe óra ipú rriarr ('pone huevo en árbol pequeño'), lacálha iafáre ('toca suelo'), ipúco ifáparr ('nace por huevo'), lacánifá rifáque ('vuela bajo'), iporétenh ('canta'), maráláfarinháfa iporétenh ('canta bonito'), iporétecá carrtaúni ripáye ('canta y se escucha a lo lejos'), cumáco iporétenh ('canta temprano'), tóji tuéta iporétenh ('canta al atardecer'), ipítenhé tójic ('sale de día'), ipítenhé tóji tuéta ('sale al atardecer'). quilhúqui/córa cúru rricanh ('come saltamontes / frutas'), malac ('comestible').

(vii) pocóronh (phasianidae) ('chirrascuá. codorniz')

Este genérico presenta un específico típico. cuyo denominador es pocóronh, polisémico en relación con el genérico y posee los siguientes rasgos: R.C. + lhacá tené icuinhca ('alas cortas'), síjafá icuinhca rriuránhe ('mueve el ala despacio'). iocá nocóchánhe ('pico corto'), iocá pilíchu inhánhe ('pico puntiagudo'), ilhóqui chá nocóchánhe ('pata pequeña'), ilhóqui chá lhacá tenénhe ('pata corta'), ilhóqui chá chacárra inhánhe ('pata como de gallina'), ámpenhé ilhóqui lenh ('garra'), ituquí lhacá tenénhe ('cola corta'), nocóchánhe óra ifilhi ('ojos pequeños'), ilhorrórro anh ('tiene cresta'), ichá quírra lhacátenénhe ('pescuezo corto'), uchúli inhánhe ('negro'), turúco ipunh ('vive en la montaña'). córa nocóchánhe óra ipú rriarr ('pone huevo en árbol pequeño'), lacálha iafáre ('toca suelo'), ipúco ifáparr ('nace por huevo'), lacánifá rifáque ('vuela bajo'), iporétenh ('canta'), maráláfarinháfa iporétenh ('canta bonito'), capífa iporétenh ('canta fuerte'), iporétecá carrtaúni ripáye ('canta y se escucha a lo lejos'), tóji tuéta iporétenh ('canta al atardecer'), ipítenhé tóji tuéta ('sale al atardecer'). quilhúqui rricanh ('come saltamontes'), malac ('comestible'). 


\subsection{Las especies}

A continuación se dará un detalle de las especies incluidas en los genéricos mencionados anteriormente, lográndose hacer un análisis de mayor inclusividad a un grado de menor inclusividad, dentro del estudio taxonómico ornitológico del guatuso.

La cantidad de especies en cada genérico varía de mayor a menor número sin que exista un parámetro que los rija. Además, es importante aclarar que el término guatuso, de acuerdo con la taxonomía científica, se puede traducir por dos o más especies. Esto implica que el informante no diferencia entre una especie u otra, presentándose un caso de infradiferenciación. Sin embargo, esto no altera o impide que dichas especies sean parte de un adecuado marco de inclusividad.

La presentación de dichas especies se hará siguiendo la teoría de Berlin, la cual denota la existencia de un taxón genérico que está formado por un conjunto de especies, donde una es la más reconocida o difundida ${ }^{11}$. Este taxón es dividido en un específico típico, el cual es polisémico con el genérico superordinario. Por otra parte, este genérico está también dividido en específicos no típicos, taxas no específicas dentro de un genérico. Estas comparten rasgos del genérico y, a su vez, se componen de uno o más rasgos atributivos.

De los cincuenta y cinco nombres genéricos que forman la taxonomía, cuarenta y siete comprenden un genérico que es polisémico con su específico típico. No se reconocen específicos no típicos. Los ocho restantes son genéricos polisémicos con un específico típico y uno o varios específicos no típicos. El genérico nhiscacá ('pajarito') tiene cuatro específicos no típicos que incluyen subclases.

Seguidamente se enumeran las especies incluidas en cada genérico, las respectivas familias y nombres científicos, además de su correspondiente en español.

11. Brent Berlin, "Speculations on the Growth of Ethnobotanical Nomenclature" (1972), en Sociocultural dimensions of language change, B. Blount y Mary Sanches, eds. (Nueva York: Academic Press, 1977) 70-101. 


\section{(i) Genérico Táro}

Específico típico

Táro

Fulica americana

Gallinula chloropus

Podilymbus podiceps

Tachybaptus dominicus

Aythya affinis

Aythya collaris

Oxyura dominica

Heliornis fulica

Anas acuta

Anas clypeata

Anas discors

Pato de agua

Pato de agua

Pato de agua

Pato de agua

Pato de agua

Pato de agua

Pato de agua

Pato de agua

Pato

Pato

Pato zarcillo

Específico

no típico Táro (suírra)

Cairina moschata

Pato real

(ii) Genérico Macmac

Específico

típico

Macmac

Cochlearius cochlearius

Cuaca

(iii) Genérico Chólechóle Específico típico

\section{Chólechóle}

Anhinga anhinga

Phalacrocorax olivaceus
Pato aguja

Pato chancho 
(iv) Genérico Pílil

Específico

típico

\section{Pílil}

Jacana spinosa

Dendrocygna autumnalis

Gallito de agua

Tryngites subrufficollis

Piche

Zarceta

(v) Genérico Túli

Específico

típico

\section{Túli}

Columbina inca

Tortolita colilar-

Leptotila verreauxi ga, San Juan

Coliblanca

Específico

no típico

\section{Caracche túli}

Claravis mondetoura

Tortolita serranera

Zenaida asiatica

Zenaida macroura

Paloma aliblanca

Geotrygon violacea

Paloma rabuda

Paloma-perdiz violácea

\section{Específico} no típico

\section{Tafá túli}

Leptotila rufaxilla

Leptotila cassinii

Paloma coronigrís Paloma pechigrís

Geotrygon lawrencii

Geotrygon veraguensis

Paloma-perdiz sombría

Paloma-perdiz bigotiblanca 
Específico no típico

\section{Cunh-óra / cunh}

Columba cayennensis

Geotrygon montana

Columba leucocephala
Paloma morada Paloma-perdiz rojiza

Paloma coroniblanca

(vi) Genérico Túfi

Específico típico

\section{Túfi}

Crax rubra

Pavón grande

(vii) Genérico Pocóronh

Específico típico

\section{Pocóronh}

Odontophorus leucolaemus

Odontophorus erythrops

Colinus leucopogon

Dendrortyx leucophrys

\section{Conclusiones}

En el estudio realizado se ha podido observar que el modelo biotaxonómico propuesto por Berlin, se cumple casi en su totalidad en el análisis biotaxonómico de las aves en guatuso.

Entre los principios básicos de jerarquización, el guatuso evidencia que existe un principio único (unique beginner), el cual presenta una división en dos ámbitos, señalados en 1.1. Esta división es la razón por la cual no se cumple el modelo biotaxonómico en su totalidad. Además, se presentan las formas de vida mayor (major lifeforms), las taxas genéricas y sus específicos, así como una taxa intermedia. No obstante, no se presentan taxas que correspondan a nombres de variedades (varietal names). 
También es importante enf atizar que en el total de rubros léxicos contenidos en la etnoornitología del guatuso, se dan órdenes de relación denotativa en relación con las taxas específicas establecidas por la taxonomía científica. Estos órdenes están formados por:

a. la correspondencia "uno a uno", y

b. la denotación infradiferenciada. denotativo:

La correspondencia "uno a uno" presenta casos en el orden

Genérico

Óla

Específico

típico

Óla

Ara macao (lapa colorada) (Psittacidae)

Específico no

típico

Aféla

Ara ambigua (lapa verde)

En cuanto a la denotación infradiferenciada, se debe mencionar la correspondencia de un rubro léxico de la lengua en cuestión, con dos o más especies establecidas por la taxonomía científica. Esto se manifiesta en la mayoría de los casos analizados. Un ejemplo de esta denotación es:

Genérico Purúnhuénhué

Específico

típico Purúnhuénhué

Cotinga ridgwayi Cotinga turquesa 
Cotinga amabilis

Procnias tricarunculata

Cyanerpes lucidus

Cyanerpes cyaneus

Dacnis cayana

Dacnis venusta

Tangara florida

Tangara icterocephala

Chlorophanes spiza

Tangara guttata

Tangara gyrola

Tangara lavinia

Tangara inornata

Tangara dowii

Tangara larvata

Chrysothlypis chrysomelas

Thraupis episcopus
Cotinga linda

Campanero tricarunculado

Picudos

Picudos

Picudos

Picudos

Tangara orejinegra

Tangara dorada

Reina

Tangara moteada

Tangaracabecicastaña

Tangara alirrufa

Tangara cenicienta

Tangara vientricastaña

Sietecolores

Tangara negro y dorado

Viuda

Finalmente, no hay evidencia de la denotación supradiferenciada, la correspondencia de dos o más rubros léxicos de la lengua en relación con una especie establecida por la taxonomía científica. 